\title{
A Proposed De-noising Algorithm
}

\author{
Nawafil Abdulwahab, Imad Al Shaikhli \\ Dept of Compute Science, International Islamic University Malaysia, Kuala Lumpur, Malaysia \\ nawafil.ali@gmail.com,imadf@iium.edu.my
}

\begin{abstract}
Minimizing noises from images to restore it and increase its quality is a crucial step. For this, an efficient algorithm was proposed to remove noises such as (salt pepper, Gaussian, and speckle) noises from grayscale images. The algorithm did that by selecting a window measuring $3 \times 3$ as the center of processing pixels, other algorithms did that by using median filter (MF), adopted median filter (AMF), adopted weighted filter (AWF), and the adopted weighted median filter (AWMF). The results showed that the proposed algorithm compared to previous algorithms by having a better signal-to-noise ratio (PSNR).
\end{abstract}

Keywords_-Gaussian noise; Salt and Pepper noise; Speckle noise; Proposed algorithm

\section{INTRODUCCTION}

Digital images, or picture elements, consist of a set of digital values called pixels, a 2-D function that can be defined as $f(x, y)$, where $x, y$ are the spatial coordinates representing the brightness and color of the image, respectively. At that point, the intensity or gray level of the image at any pair of coordinates is called the amplitude of $f$. A 1-bit is defined as an array of numbers between $0-255$ [1]. Digital images degraded by noises, especially during transmission via electronic communication devices, result in disturbed, blurred, and clouded artifacts on the images. Image restoration is a technique that can remove the abovementioned noises while also preserving the quality of the image (as much as possible) [2, 3].

Researchers are working on developing de-noising techniques for improving digital images over the past two decades. The most critical image restoration aspect is removing or reducing unwanted pixels and preserving the original images [4].

This paper is organized per the following: section one introduces the paper; section two discusses the literature on the topic, section three details the types of image noises. Section four describes de-noising algorithms used to develop the proposed algorithms, section five details the proposed algorithm, section six outlines the simulation and performance evaluation, and section seven presents the experimental results and comparisons. Section eight discusses the results, and section nine concludes the paper.

\section{LITERATURE REVIEW}

Many image de-noising techniques were developed to improve digital images over the past two decades. [6] simulated the addition of noise algorithm to evaluate the effect of implementing noise variations of $20-70 \%$ using various window sizes on grayscale and RGB images. They applied the median filter for detecting noises and eliminated deterioration in the image. The experimental results and the performance of the median algorithm were analyzed per the effects of noise removal from the image at a $3 \times 3$ window size, and they reported that the performance of their algorithm is better than that of window size of $9 \times 9$ and low noise variance [8].

[22] proposed the (ADBUTMF) algorithm for removing noises from degraded images. They reported that the ADBUTMF algorithm performed better in terms of removing noise from corrupted images relative to the Modified Decision Based Algorithm (MDBA), Median Filter (MF), Modified Decision Based Unsymmetric Trimmed Median Filter (MDBUTMF), Decision Based Algorithm (DBA), and Progressive Switched Median Filter (PSMF) algorithms [9] [10]. They tested their proposed algorithm on many grayscale and color images and reported that they obtained better PSNR and IEF, especially at higher noise densities of up to $\sim 80-90 \%$.

[25] proposed a modified decision based median filter for noise removal from corrupted grayscale and color images. Comparison with existing algorithms such as Adaptive Median Filter (AMF), Decision Based Median Filter (DBMF), Switching Median Filter (SMF), and Modified Decision Based Median Filter (MDBMF) were conducted. Modified decisions based median algorithm were used to enhance the image for further processing using image processing operations [24]. The performance evaluation of the proposed algorithm confirmed that compared with other methods such as AMF, SMF, and DBA, the former performs better in terms of the PSNR, while (DBMF) reported a lower PSNR value in the case of noisy images. They suggested that future works use nonlinear filters such as (MNF) to modify and remove noise from images based on quantitative parameters such as PSNR and MSE.

[27] proposed combined filter structures of the median, adaptive median, and weighted median for removing noises from degraded images. They used the 
median algorithm, which is nonlinear and suitable for small window sizes such as $3 \times 3$ at low noise densities, where when the window sizes increased, the pixels will be damaged and blurred at higher noise densities. They used the adaptive median algorithm to track the corrupted pixels while excluding the undamaged pixels. Each algorithm has it significant use the most important window size at high-level noises is performed through the adaptive median. The best algorithm for removing high noise levels from grayscale and color images is the weighted median algorithm. Their results confirmed that the performance evaluation of SMF and AMF is comparable to other noise removal algorithms based on the PSNR value.

[28] discussed and compared various noise types and noise removals techniques, such as the adaptive median filter (AMF), simple adaptive median filters (SAMF), and median filter (MF). Combining median filters with other filters resulted in better PSNR. The PSNR of SAMF increased in high noise density values but decreased when dealing with low noise density values. Similarly, MF and AMF reported higher PSNR relative to that of the SAMF.

[21] proposed an efficient (new) Modified Mean-Median filter for removing noise from damaged images, and then compared it to techniques such as mean filter (MF), meanmedian filter (MMF), median filter(MF), super mean filter (SUMF), weighted median filter (WMF), decision-based median filter (DBMF), and the standard median filter (SMF). WMF preserves the edges in high noise variances. Each filter has its respective advantages relative to SUMF in the context of removing high noise density from images. SMF and DBMF were used to remove impulse noise. The discontinuity in the underlying regression function was done using MF, while DBMF was used for de-noising at lower levels. The (new) WMF was introduced to overcome the previously mentioned problems associated with these techniques, and it performed well in decreasing high-level noises as it preserves most of the edges of the images.

[23] proposed an adaptive median algorithm for removing noises from corrupted grayscale images. The adaptive median filter requires two steps: first, the image's pixels are determined using degraded noise by increasing the window size automatically until it identifies the corrected pixel degraded by the noise, then move on to substitute it with a suitable median value. The method employs different types and sizes of grayscale images at various noise variances. They confirmed that the proposed algorithm is superior based on the results obtained when compared with other algorithms such as standard median filter (SMF), switching mean median filter (SMMF), weighted median filter (WMF), which were evaluated with Mean square error (MSE), Structural Similarity Index (SSIM), and peak signal-to-noise ratio (PSNR) parameters. They proposed that future works on the subject involve combining combined algorithms for improving noise removal technology.

[26] proposed an efficient algorithm called the adaptive median algorithm, which removes noises from color and grayscale images with high noise variance of up to $90 \%$ with impulse noises while preserving details and enhancing image quality. Their method selects suitable neighborhood values to obtain a specific median at each $3 \times 3$ window using the signal filtration overlapping window. The adaptive median algorithm's effectiveness exceeded the MSE, PSNR, IEF, RMSE Time, and SSIM per their respective quantitative image metrics. It's also relatively better than VMF, DBA, SMF, and ROAMF. The adaptive median algorithm used $a 3 \times 3$ fixed and small window size, rendering it faster than the ROAMF. MATLAB was used to evaluate the proposed active median filter.

\section{IMAGE NOISE}

Noise can be identified as a random intensity variation present in an image during the image transmission processing or acquisition coding steps. It results in undesirable artifacts, such as unseen lines corners, unrealistic edges, and blurred objects, while disturbing background scenes.

\section{A. Gaussian noise}

Gaussian noise can be additive or subtractive based on fluctuations in the detectors or amplifiers. It generally disrupts the greyscale values in digital images and natural sources, such as the discrete nature of radiation of warm objects and atoms' thermal vibration. It is also the main factor of the normalizing histogram for gray value or design and characteristics via its PDF. The additive white is created by Gaussian noise, which results from electronic error, and can be removed from an image using the proposed adaptive filter called the probability density function (PDF) [9-11].

\section{B. Salt and pepper}

Salt and pepper noise compares pixels to its neighbors based on its colors and intensity values. It can also be defined as impulse noise, random noise, or independent noise. It is created from unexpected changes in images. Random noises usually replace pixel values to its minimum or maximum permissible values, which corrupts the grayscale image. White spots are called salt, while black spots are called pepper in an image [12] [13].

\section{Speckle noises}

Speckle noises occur in active radar, medical ultrasound, optical coherence tomography images, medical ultrasound, and synthetic aperture radar. It degrades the quality of an image. Speckle noise can also be called the multiplicative 
noise of the image pixel values as the multiplications of random values can determine it. Speckle noise can be removed from images using adaptive and non-adaptive filters, with the mean filter method being the best technique for doing so [14] [15].

\section{IMAGE DE-NOISE ALGORITHMS}

Image noise reduction techniques are essential for image analyses. The primary objective of image restoration is to remove noises from an image while preserving its original features. Removing undesirable noises from an image is the main objective of filtering and is critical towards restoration and enhanced image processing. The best filtering algorithm can remove noises, smooth and sharpen images, and detect edges without compromising the image itself.

\section{A. Median filter}

The median filter is typical in image processing and is classified as a nonlinear filter technique. It is regularly used to remove salt and pepper and speckle noises from images and other types of signals from corrupted images. The median filter works well in low noise densities. However, when used for modified images, both noise pixels and noise pixels are restored. The advantage of using a median filter is that it works well on blurred, thinning, or corner distortions in images in low noise densities while producing unpreserved edge details in higher noise densities close to the original image [16-19].

The proposed algorithm is a step-by-step approach; Step 1: pinpoint corrupted pixels in an image; if corruption is salt and pepper, if $1 \geq$ pixel $\geq 254$, it will remain unchanged; otherwise, the intensity value of pixels from o or 255 will decrease. Step 2: set a window size of $3 \times 3$ and make $x, y$ the window's center pixels. Step 3: convert the 2D image into 1D, containing 9 elements in a $3 \times 3$ window. Step 4: create an array with ascending rows. Step 5: take the median of a single row element; if $\mathrm{n}$ is an odd value, then the Median $=(n+1) / 2^{\text {th }}$ term of the observation; if $n$ is even, then Median $=$ mean of term $(n / 2) 2^{\text {th }}$ and term $=(n+1) / 2^{\text {th }}$ of the given observation. Step 6: replace the center pixel median value. Step 7: use the same process for all noisy and noisefree pixels. Step 8: end.

\section{B. Weighted Median Filter}

The Weighted median filter (WMF) and standard median filter (SMF) are quite similar. However, the former has a weight component, and each of its filter element requires the duplications of its corresponding weights number that is controlled by the median value [20] [13] [15].

\section{Adaptive Median Filter}

The adaptive median filter (AMF) is similar to standard median filters (SMF), as both are advance nonlinear filters.
The AMF pinpoints noise-corrupted pixels. The AMF matches every pixel with its neighbors after distinguishing the corrupted and uncorrupted ones within the image. The AMF performs well [21][23]. The proposed algorithm's steps are similar to the median filter up till step 7 , which is repeating the same process by increasing the window size if it meets the specific conditions of $5 \times 5,7 \times 7$, and $9 \times 9$, and if the process meets the satisfactory condition, the pixels will use the median of the window. This is followed by Step 8: repeat the same process for all noise and noise-free pixels, and Step 9: end.

\section{Decision based Adaptive Median Filter}

The Decision Based Algorithm (DBA) identifies the noisy pixel by assigning the intensity values with a minimum or maximum (0-255, 8-bit image). This is done by determining the window or neighborhood values and replacing the noisy pixel value with median/mean/mid-point values. In high noise density, DBA decreased noise density by 30-40\% [22] [24]. The algorithm starts with Step 1: identify the 2D image and create a $3 \times 3$ window size for pixel analysis. Step 2: convert the $2 \mathrm{D}$ image into $1 \mathrm{D}$ in a single row. Step 3: create a condition statement; if pixel $=0,255$, then yes, will eliminate and select all the o's and 255's; when it is no, the DBA arranges the remaining elements in ascending order. Step 4: DBA will select the median in the grayscale image. Step 5: replace the center pixel with the median value. Step 7: repeat step 6 by increasing the window size to $5 \times 5,7 \times 7$, and 9x9. Step 8: end.

\section{E. Modified decision based Unsymmetric Median Filter}

Modified decision-based Unsymmetric Median Filter identifies noise pixel by replacing it using the Trimmed Median Filter (TMF) [23][26]. The proposed algorithm represents pixel values with o's and 255's in the window and removes noises from the image in a high noise variance of $\sim 70-90 \%$. The proposed algorithm starts with Step 1: select a 2-D window with the size $3 \times 3$. Step 2: when the pixel is processed, the pixels left unchanged are the uncorrupted pixel. Step 3: when eliminating elements with values o's and 255's after selecting a window, the determined corrupted pixel means two possible cases, where i) the mean will replace the element of the window, and ii) when the selected window elements do not contain o's and 255's, then the remaining elements will search for the median value. Step 4: Repeat steps 1 to 3 until the pixels in the entire image are processed.

\section{THE PROPOSED ALGORITHM}

This section describes the proposed algorithm that enhances the performance and overcomes the drawbacks of fixed window size. The simple version of the SMF was adapted and modified for the proposed algorithm 
First, a standard color image was selected, then a $3 \times 3$ window was created, and the center pixel affixed as the processed pixel. Every value was searched at the designated window size, and when it found a corrupted pixel due to noise, it is replaced with the median. The same process was used on the column with an expanded window size until if found a corrupted pixel, then the processing pixel proceeded to preserve its value. The proposed algorithm works as follows:

Let, $\mathrm{W}$ is the window size

$W_{\text {mas }}$ is the maximum window size

$\mathrm{W}_{\min }$ is the minimum window size

$P(i, j)$

\begin{tabular}{|c|c|}
\hline Input: & RGB digitized painting Image \\
\hline Output: & Enhanced image mask \\
\hline \multicolumn{2}{|l|}{ Begin } \\
\hline Step1: & $\begin{array}{l}\text { Define a } 3 \times 3 W(i, j) \text { whch is the mask of pixel } \\
\text { value } p(i, j)\end{array}$ \\
\hline Step2: & $\begin{array}{l}\text { Define an array arraymedianrow [median } \\
\text { (First row of mask), (second row of mask), } \\
\text { (n row of mask)]; }\end{array}$ \\
\hline Step3: & $\begin{array}{l}\text { Define an array arraymediancolumn } \\
\text { [median (first column of mask), (second } \\
\text { column of mask), ( } \mathrm{n} \text { column of mask)]; }\end{array}$ \\
\hline Step4: & medianrowelm=median (arraymedianrow); \\
\hline Step5: & $\begin{array}{l}\text { medianColElm=median } \\
\text { (arraymediacolumn); } \\
f(x, y)=\text { median(medrowselm, medcolselm, } \\
f(x, y))\end{array}$ \\
\hline Step6: & $\begin{array}{l}\text { Repeat step4: and step5: for all images in } \\
\text { dataset. }\end{array}$ \\
\hline Step7: & Compute MSE and PSNR \\
\hline Step8: & End \\
\hline
\end{tabular}

\section{SIMULATION AND PERFORMANCE EVALEATION}

The simulation was continued using nine standard color images with different formats and variances. These images were corrupted with Gaussian, salt and pepper, and Speckle noises. The proposed algorithm was used to remove the noise from the grayscale images.

The performance of the proposed approach was evaluated using MATLAB (2016) using the parameters MSE and PSNR calculated using equation (2):

$$
\text { PSNR }=10 \log 10\left(\frac{255^{2}}{M S E}\right)
$$

Where 255 is the maximum value of the pixel present in an image and MSE is the mean square error between the original and de-noised image with the size $M * N$. MSE is defined per equation (2):

$$
M S E:=\frac{1}{m n} \sum_{i=1}^{m} \sum_{j=1}^{n}(e(i, j)-f(i, j)
$$

Where $\mathrm{e}(\mathrm{i}, \mathrm{j})$ is the earliest form of the original image, and $f(i, j)$ is the final form of the de-noised image[25].

\section{EXPEREMENTAL RESULTS AND COMPARISON}

For the simulation studies, the comparisons are shown in Tables 1 \& 3, where image1 was degraded with salt and pepper, Gaussian, and Speckle noises and compared with the proposed algorithm and other noise removal algorithms such as the MF, AMF, AWF, and AWMF. The simulation was implemented in nine digital images, with an example shown below:

TABLE I

COMPARITIVE PSNR FOR PROPOSED ALGORITHM AND EXESTING ALGORITHMS WITH GAUSSIAN NOISE

\begin{tabular}{|c|c|c|c|c|c|}
\hline \multirow{2}{*}{$\begin{array}{c}\text { Noise } \\
\text { Variance }\end{array}$} & MF & AMF & AWF & AWMF & PA \\
\cline { 2 - 6 } & \multicolumn{5}{|c|}{ PSNR } \\
\hline 10 & 24.21 & 33.14 & 36.31 & 36.28 & 39.96 \\
\hline 20 & 24.46 & 30.93 & 32.92 & 33.75 & 37.58 \\
\hline 30 & 24.25 & 29.42 & 33.64 & 31.35 & 35.36 \\
\hline 40 & 24.05 & 27.21 & 32.97 & 30.37 & 33.68 \\
\hline 50 & 23.72 & 29.08 & 30.13 & 31.68 & 32.43 \\
\hline 60 & 22.51 & 26.08 & 30.75 & 30.47 & 30.72 \\
\hline 70 & 20.44 & 25.37 & 28.18 & 29.21 & 29.47 \\
\hline 80 & 18.22 & 22.64 & 26.74 & 27.64 & 27.83 \\
\hline 90 & 16.79 & 20.01 & 24.94 & 25.91 & 26.92 \\
\hline
\end{tabular}

TABLE II

COMPARITIVE PSNR FOR PROPOSED ALGORITHM AND EXESTING ALGORITHMS WITH SALT \& PEPPER NOISE

\begin{tabular}{|c|c|c|c|c|c|}
\hline \multirow{2}{*}{$\begin{array}{c}\text { Noise } \\
\text { Variance }\end{array}$} & MF & AMF & AWF & AWMF & PA \\
\cline { 2 - 6 } & \multicolumn{5}{|c|}{ PSNR } \\
\hline 10 & 22.21 & 33.14 & 34.31 & 34.28 & 39.28 \\
\hline 20 & 22.46 & 30.93 & 32.92 & 32.75 & 39.75 \\
\hline 30 & 22.25 & 29.42 & 33.64 & 31.35 & 39.35 \\
\hline 40 & 22.05 & 27.11 & 32.97 & 30.37 & 39.37 \\
\hline 50 & 19.72 & 29.08 & 30.13 & 31.68 & 39.68 \\
\hline 60 & 19.51 & 26.08 & 24.75 & 28.47 & 37.47 \\
\hline 70 & 20.44 & 25.17 & 28.18 & 29.01 & 37.01 \\
\hline 80 & 18.22 & 22.64 & 26.74 & 27.64 & 33.64 \\
\hline 90 & 16.79 & 20.01 & 24.94 & 25.91 & 32.91 \\
\hline
\end{tabular}


TABLE III COMPARITIVE PSNR FOR PROPOSED ALGORITHM AND EXESTING ALGORITHMS WITH SPECKEL NOISE

\begin{tabular}{|c|c|c|c|c|c|}
\hline $\begin{array}{c}\text { Noise } \\
\text { Variance }\end{array}$ & MF & AMF & $\begin{array}{c}\text { AWF } \\
\text { PSNR }\end{array}$ & AWMF & PA \\
\hline 10 & 20.68 & 24.54 & 28.56 & 35.87 & 39.87 \\
\hline 20 & 21.46 & 23.87 & 28.45 & 35.34 & 39.45 \\
\hline 30 & 21.45 & 22.65 & 27.56 & 35.52 & 39.23 \\
\hline 40 & 21.67 & 22.56 & 26.67 & 35.87 & 38.23 \\
\hline 50 & 21.87 & 22.34 & 26.35 & 35.87 & 37.67 \\
\hline 60 & 20.65 & 23.46 & 25.67 & 35.43 & 35.87 \\
\hline 70 & 19.45 & 23.78 & 26.87 & 32.43 & 33.88 \\
\hline 80 & 19.76 & 23.65 & 25.45 & 31.76 & 33.73 \\
\hline 90 & 19.45 & 23.86 & 25.88 & 32.45 & 33.84 \\
\hline
\end{tabular}

The graph of figure 1 to 3, illustrates the PSNR values of the comparisons of proposed algorithm and related work, as shows bellow:

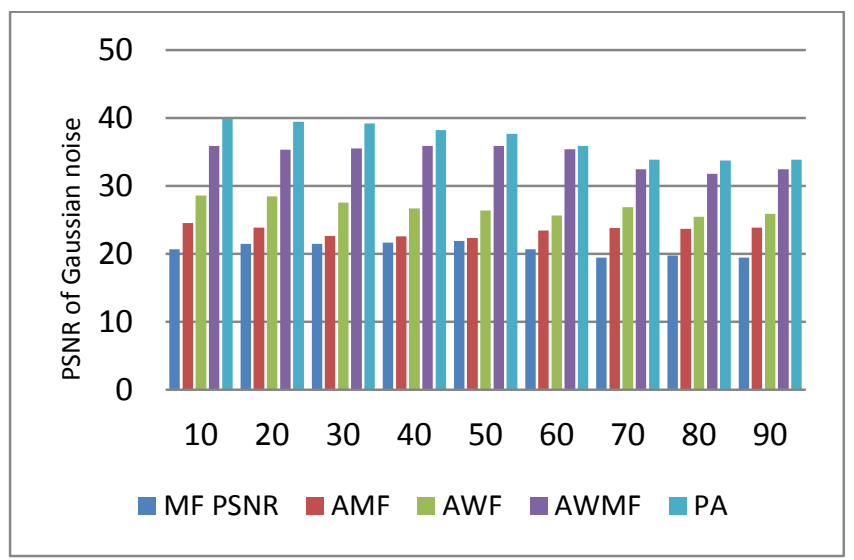

Fig. 1 PSNR with proposed algorithm at different noise level corrupted with Gaussian level

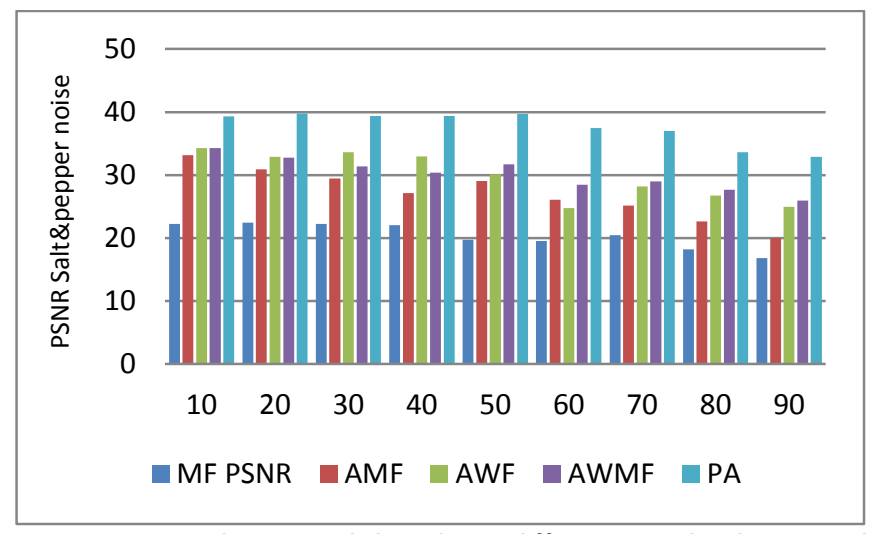

Fig.2 PSNR with proposed algorithm at different noise level corrupted with salt and pepper level

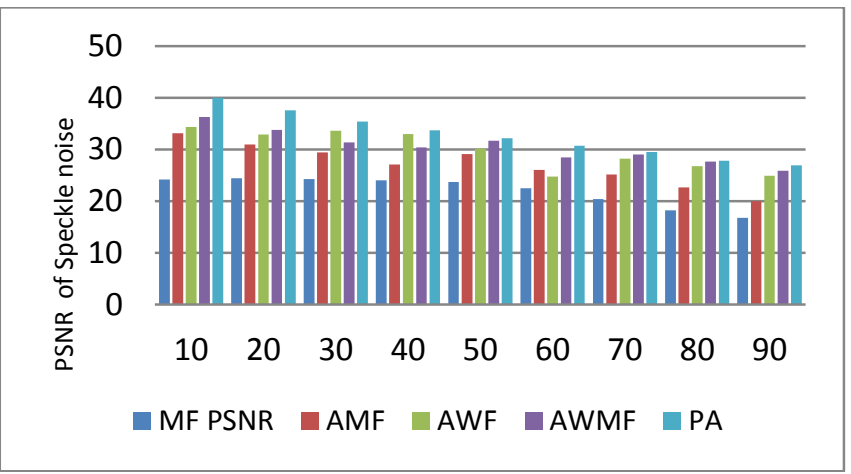

Fig.3 PSNR with proposed algorithm at different noise level corrupted with Speckle noise

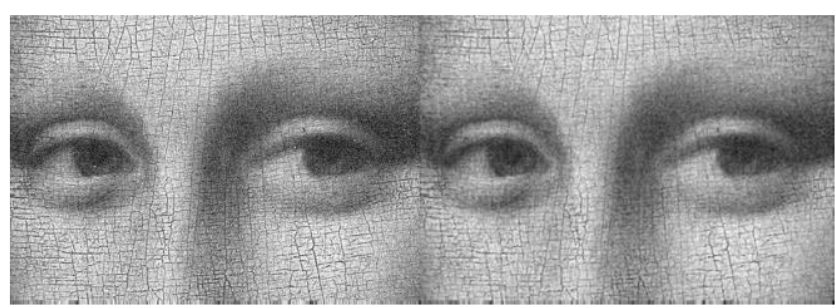

Fig.4 Performance of proposed algorithm corrupted with $30 \%$ Gaussian noise

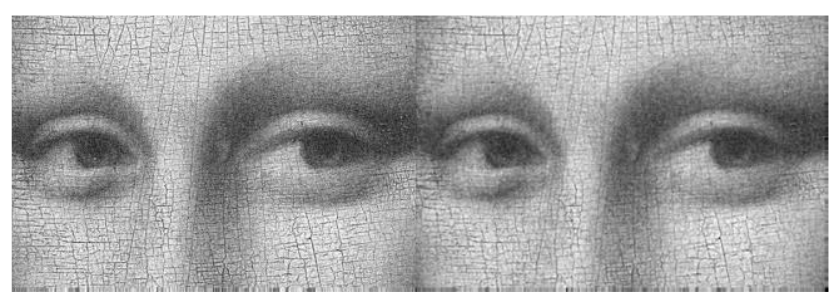

Fig.5 Performance of proposed algorithm corrupted with $60 \%$ Gaussian noise

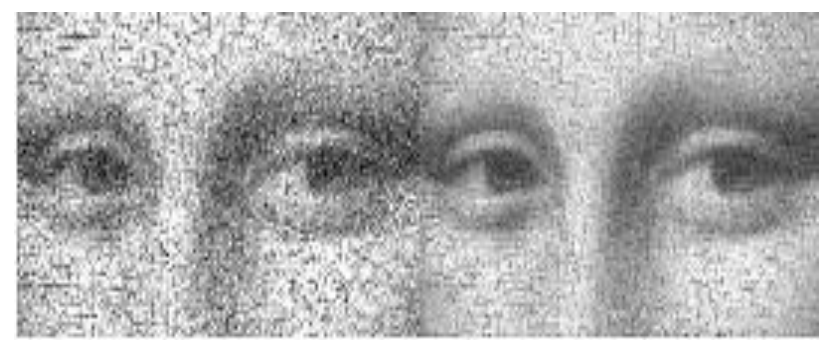

Fig.6 Performance of proposed algorithm corrupted with 90\% Gaussian noise

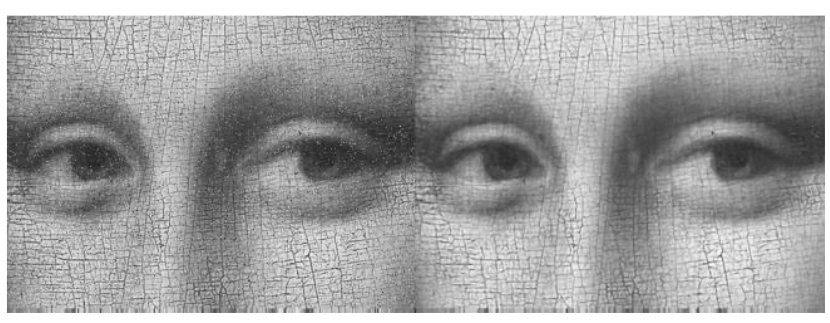

Fig.7 Performance of proposed algorithm corrupted with 30\% Salt\& Pepper Noise 


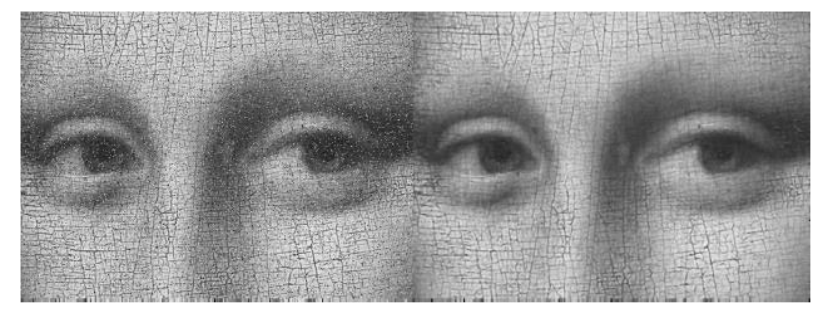

Fig.8 Performance of proposed algorithm corrupted with $60 \%$ Salt\& Pepper Noise

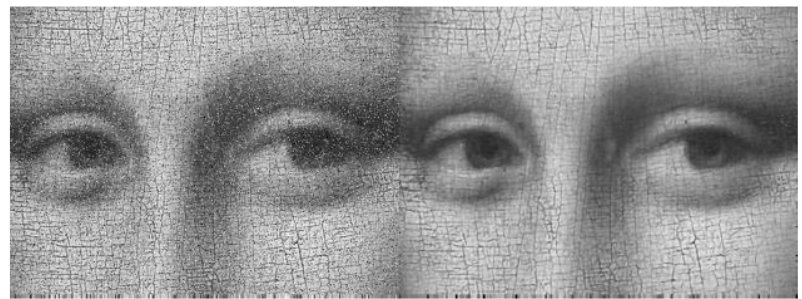

Fig.9 Performance of proposed algorithm corrupted with 90\% Salt\& Pepper Noise

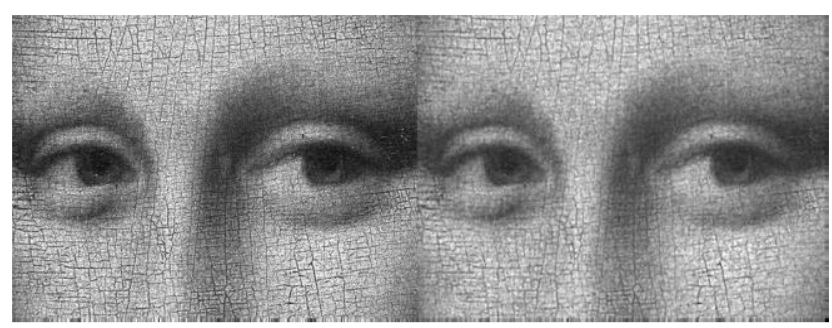

Fig.10 Performance of proposed algorithm corrupted with 10\% Speckle Noise

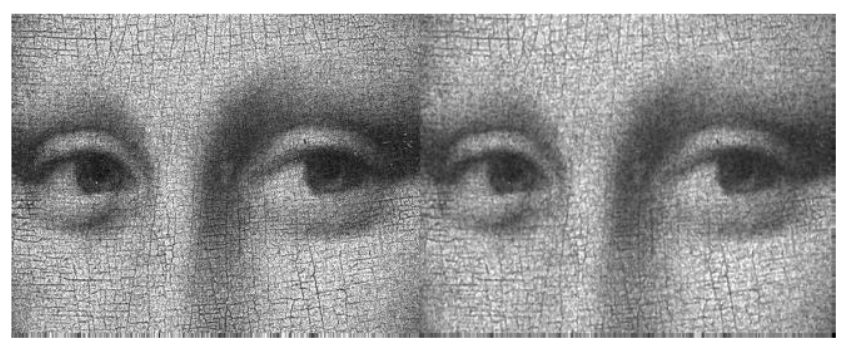

Fig.11 Performance of proposed algorithm corrupted with $60 \%$ Speckle Noise

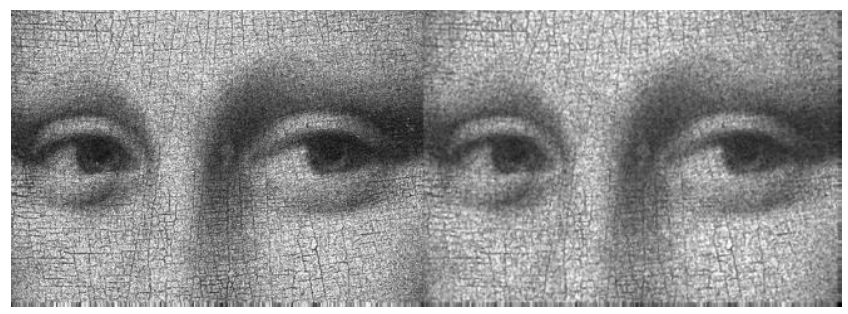

Fig.12 Performance of proposed algorithm corrupted with 90\% Speckle Noise

\section{DESCUSSION}

This section discusses the results obtained from the proposed algorithm relative to that obtained from other algorithms when dealing with an image corrupted by
Gaussian, Speckle, and salt and pepper noises. Different filtering techniques were developed for removing noises from images in order to improve its quality.

Figure 1 shows the graphical comparison of the proposed algorithm and other algorithms in the form of image1, where the corruption caused by Gaussian noise at different noise levels extends from $10 \%$ to up to $90 \%$. The PSNR value of the proposed algorithm is the highest, and its performance in noise removal exceeds that of the other algorithms.

Figure 2 shows the corruption of image 1 due to the presence of salt and pepper noise. The proposed algorithm was used to remove the noise from $10 \%$ to up to $90 \%$. The PSNR values confirmed that the performance of the proposed algorithm exceeded that of the others.

Figure 3 shows graphs of image 1 corrupted by Speckle noise. The proposed algorithm was used to remove the noise from the image. The PSNR values reported by other algorithms is lower relative to that of the proposed algorithm.

It is evident from Figure $4-11$ that the proposed algorithm performed better at de-noising at low and high noises at 30\%,60\%, and 90\% for the Gaussian noise, salt, and pepper noise, and Speckle noise, respectively.

Based on the results, the proposed algorithm's high PSNR values relative to the other algorithms render it suitable for noise removal in images degraded by high noise levels. It also preserves edges and smooths the image in order to preserve its quality.

\section{CONCLUSION}

This work analyzed proposed algorithms developed for remove noises from images that corrupted by Gaussian, salt and pepper, and Speckle noises at multiple levels. A comparison between the proposed algorithms and other algorithms were conducted by decreasing the number of removed pixels from an image, where the performance evaluation confirmed that the proposed algorithm performed better results in removing noise than the others based on its higher PSNR values on a grayscale image, noise removal indicates smoothed and edge preservation in an image.

\section{REFERENCES}

[1] I. F. T. A.-S. Nawafil Abdulwahab, Raini Hasan "Detection of Cracked Digitized Paintings and Manuscripts Based on Threshold Techniques " Journal of Computational and Theoretical Nanoscience, , vol. 16, , p. 7, 2019.

[2] N. A. Ali and I. F. T. Al Shaikhli, "Detection and Restoration of Cracked Digitized Paintings and Manuscripts using Image Processing-A Survey," International Journal on Perceptive and Cognitive Computing, vol. 3, 2017.

[3] I. F. T. A.-S. Nawafil Abdulwahab Farajalla Ali, Raini Hasan "Detection And Restoration of Cracked Digitized Paintings and Manuscripts Using 
Image Processing " International Journal of Engineering \& Technology, vol. 7 p. 5, 2018

[4] Z. Gao, "An Adaptive Median Filtering of Salt and Pepper Noise based on Local Pixel Distribution " International Conference on Transportation \& Logistics, Information \& Communication, Smart City p. 11, 2018.

[5] F. M. Abubakar, "Study of Image Segmentation using ThresholdingTechnique on a Noisy Image," International Journal of Science and Research (IJSR), India Online vol. 2 p. 3, 2013.

[6] P. Z. a. F. Li, "A New Adaptive Weighted Mean Filter for Removing Saltand-Pepper Noise "IEEESIGNALPROCESSINGLETTERS, vol. 21, p. 4, 2014.

[8] N. K. A. Rachna Mehta, "Comparative Analysis of Median Filter and Adaptive Filter for Impulse Noise - A Review "International Journal of Computer Applications p. 6, 2014.

[9] M. M. P. S.V.Halse2, "A Review Paper: Study of Various Types of Noises in Digital Images," International Journal of Engineering Trends and Technology vol. 57, p. 4, 2018

[10] A. P. Mauro Barni, and Alessandro Piva, "Image Processing for the Analysis and Conservation of Paintings: Opportunities and Challenges," IEEE SIGNAL PROCESSING MAGAZINE, vol. VOL. 22, NO. 5, , pp. 1-4, 2005

[11] P. A. B. Sakshi Tiwari1, Prof. Raj Kumar Paul3 "Comparative Performance Analysis of SALT and PEPPER Noise Removal " Journal of Computer Engineering (IOSR-JCE) vol. 16, p. 6, 2014.

[12] D. M. Hanumantharaju, M. Ravishankar, D. R Rameshbabu, and M. Aradhya, A Novel Full-Reference Color Image Quality Assessment Based on Energy Computation in the Wavelet Domain vol. 22, 2013

[13] F. Abas and K. Martinez, "Craquelure analysis for content-based retrieval," in Digital Signal Processing, 2002. DSP 2002. 2002 14th International Conference on, 2002, pp. 111-114.

[14] P. B. Dasgupta, "Analytical Comparison of Noise Reduction Filters for Image Restoration Using SNR Estimation," International Journal of Computer Trends and Technology (IJCTT) vol. volume 17 number 32014

[15] M. Barni, A. Pelagotti, and A. Piva, "Image processing for the analysis and conservation of paintings: opportunities and challenges," IEEE Signal processing magazine, vol. 22, pp. 141-144, 2005.

[16] M. O. Ahmad Odat, and Fadi Shehadeh "Image Denoising by Comprehensive Median Filter " International Journal of Applied Engineering Research vol. 0973-4562, p. 7, 2015.
[17] T. V. S. Esakkirajan, Adabala N. Subramanyam, and C. H. PremChand, "Removal of High Density Salt and Pepper NoiseThrough Modified Decision Based UnsymmetricTrimmed Median Filter," IEEE Signal processing magazine, vol. 18 p. 4, 2011.

[18] P. SnehalAmbulkar, "A Review of Decision Based Impulse Noise Removing Algorithms," Snehal Ambulkar et al Int. Journal of Engineering Research and Applications vol. Vol. 4 p. 6, 2014.

[19] S. K. S. Arabinda Dash, "High Density Noise Removal By Using Cascading Algorithms " Fifth International Conference on Advanced Computing \& Communication Technologies, p. 6, 2015

[20] B. A.Srinagesh, Dr.G.P.Saradhi Varma "A New Method for Removal of Salt and Pepper Noise through Advanced Decision Based Unsymmetric Median Filter " International Journal of Computer Science and Information Technologies,, vol. Vol. 5(6), p. 6, , 2014, .

[21] J. Lalit Kumar, Mithlesh, "Noise removal technique from digital image using advance median filter algorithm " International Journal of Advance Research, Ideas and Innovations in Technology vol. 4, p. 7, 2018.

[22] K. S. S. Saranya, "Comparative Analysis of Various Median Filters Using Peak Signal TO Noise Ratio (PSNR) " International Journal of Science, Engineering and Technology Research (IJSETR), vol. 3, p. 5, 2014.

[23] V. K. G. Brijnandan Chaturvedi "Advanced Technology for remove salt and pepper Noise from Images "International Journal of Scientific Research Engineering \& Technology, vol. 3, p. 3, 2014.

[24] T. V. S. Esakkirajan, Adabala N. Subramanyam, and C. H. PremChand, "Removal of High Density Salt and Pepper Noise Through Modified Decision Based Unsymmetric Trimmed Median Filter " IEEESIGNALPROCESSINGLETTERS, vol. 18, p. 4, 2011.

[25] S. A. Deepalakshmi R1, "Survey on Salt and Pepper Noise Remova Techniques," International Journal of Innovative Research in Science, Engineering and Technology vol. 6, p. 5, 2017.

[26] A. K. B. a. B. K. Joshi2, "A REVIEW PAPER: NOISE MODELS IN DIGITAL IMAGE PROCESSING," Signal \& Image Processing : An International Journal (SIPIJ) vol. Vol.6, , p. 63, 2015.

[27] T. A. R. S. Abdul Saleem, "An Effective Noise Adaptive Median Filter for Removing High Density Impulse Noises in Color Images," International Journal of Electrical and Computer Engineering, vol. 6, p. 10, 2016. 